\title{
Science Fiction im Werk von Friedrich Dürrenmatt
}

\author{
[Science Fiction in the Work of Friedrich Dürrenmatt] \\ http://dx.doi.org/10.11606/1982-883720321
}

\section{Valeria Sabrina Pereira ${ }^{1}$}

\begin{abstract}
Besides his plays, Friedrich Dürrenmatt was known for his incursions in the detective novel, where he challenged the limits of the genre; one of his texts is even subtitled Requiem for the Detective Novel. Less known is the fact that he also published texts inspired by science fiction, such as the radio play Das Unternehmen der Wega (1954), and the narratives "Der Winterkrieg in Tibet" (1981) and "Der Versuch" (posthumous, 1992). As per usual in his work, Dürrenmatt makes large use of irony and sarcasm, but only the radio play satirizes the genre Science Fiction itself. The narrations, in contrast, make use of the typical characteristics of the genre in order to perform the social critic intended by the author. This paper will discuss how the cited works reflect the relation of the author to science fiction and the Zukunftsroman, the 'national' version of the genre in German speaking countries until the middle of the $20^{\text {th }}$ century.
\end{abstract}

Keywords: Science Fiction; Zukunftsroman; popular fiction; apocalypse; utopia

Zusammenfassung: Neben seinen Theaterstücken ist Friedrich Dürrenmatt bekannt für seine Ausflüge in die Detektivgeschichte, bei denen der Autor mit den Grenzen des Genres spielte; dem Roman Das Versprechen gab er sogar den Untertitel Requiem auf den Kriminalroman. Weniger bekannt ist, dass er auch Texte publizierte, die von der Science Fiction inspiriert sind: das Hörspiel Das Unternehmen der Wega (1954), und zwei Erzählungen, "Der Winterkrieg in Tibet" (1981) und "Der Versuch" (posthum, 1992). Wie in seinem Werk überhaupt, bedient sich Dürrenmatt auch hier der Ironie und des Sarkasmus, aber nur im Hörspiel ist die Science Fiction selbst Ziel der Satire und impliziert eine Distanzierung vom Unterhaltungsgenre. In den Erzählungen dagegen werden die Gattung bzw. einige ihrer Eigenschaften gebraucht, um die vom Autor beabsichtigte Sozialkritik zu formulieren. In diesem Artikel wird diskutiert, wie die genannten Werke die Beziehung des Autors zur Science Fiction und zum Zukunftsroman spiegeln, der älteren deutschsprachigen Variante der Gattung, die bis zur Mitte des 20. Jahrhunderts das 'nationale' Genre bildete.

Stichwörter: Science Fiction; Zukunftsroman; Unterhaltungsliteratur; Apokalypse; Utopie

\footnotetext{
${ }^{1}$ Universidade de São Paulo, Faculdade de Filosofia, Letras e Ciências Humanas, Departamento de Letras Modernas, Av. Prof. Luciano Gualberto, 403, São Paulo, SP, 05508-010, Brasil. E-mail: valeriasabrinap@gmail.com. Esta pesquisa foi desenvolvida com apoio de bolsa de pesquisa da CAPES (Coordenação de Aperfeiçoamento de Pessoal de Nível Superior), Proc. BEX 7401/14-0.
} 


\section{Einführung $^{2}$}

Neben seinen Theaterstücken wurde Friedrich Dürrenmatt insbesondere für seine Ausflüge in den Detektivroman bekannt, mit Romanen wie Der Richter und sein Henker (1950) und Das Versprechen - Requiem auf den Kriminalroman (1958). Dies war jedoch nicht die einzige Richtung der Genreliteratur, mit der er experimentierte. Merkmale der Science Fiction können schon im Finale von Die Physiker (1962) und in der Handlung des Stücks Der Mitmacher (1976) beobachtet werden. In anderen Werken ist die Gattung voll entwickelt, so im Hörspiel Das Unternehmen der Wega (1954) und in den beiden Erzählungen "Der Winterkrieg in Tibet" (1981) und "Der Versuch" (posthum, 1992).

Die Werke Dürrenmatts wollten das Nachdenken über die Gegenwartsgesellschaft und ihre Probleme ermöglichen. In der Nachfolge Kants (EMTER 1995: 240) glaubte Dürrenmatt, dass die Realität nur mit Hilfe der Wissenschaften erkannt werden könne und dass die Literatur demnach denselben Wegen folgen müsse wie die wissenschaftliche Forschung. Dürrenmatt legte freilich den Wissenschaftsbegriff des 20. Jahrhundert zugrunde, wie sich in Von Sinn der Dichtung in unserer Zeit (1956/1966) erkennen lässt. In diesem Aufsatz weist der Autor auf die aktuelle Entwicklung wissenschaftlicher Forschung hin, die zeigt, dass die Naturwissenschaften sich immer mehr der mathematischen Abstraktion annähern müssten, um der tatsächlichen Komplexität der Welt gerecht zu werden (DÜRRENMATT 1998: 61-64). Dass sich die Literatur nicht in dieselbe Richtung entwickelt hat, wird hier kritisch gesehen. Da eine wirksame Darstellung der Wirklichkeit nicht möglich sei - so Dürrenmatts Argumentation -, solle die Literatur sich vom Realismus entfernen und sich auf das Entwerfen von möglichen Welten verlegen. ${ }^{3}$ Solche Welten könnten -

\footnotetext{
${ }^{2}$ Ich bedanke mich herzlich für die Einladung an die Universität Bern durch Oliver Lubrich und die freundliche Aufnahme durch die Mitarbeiter des Centre Dürrenmatt und des Schweizerischen Literaturarchivs Bern. Die Hinweise Ulrich Webers, der für den Nachlass Dürrenmatts verantwortlich ist, waren für diese Untersuchung besonders hilfreich.

${ }^{3}$,Dürrenmatt [...] bezieht den Stand der modernen Physik in seine erkenntnistheoretischen Überlegungen mit ein. Die Relativitäts- und die Quantentheorie haben gezeigt, daß Denkformen nur den Anschein von Notwendigkeiten erwecken. Zudem wurde deutlich, daß nicht die Natur an sich, sondern die vom Menschen befragte Natur Gegenstand der Physik ist. Dadurch wurde die erkenntnistheoretische Auseinandersetzung der Physik mit ihren Grundlagen, mit ihrem Denkgerüst erforderlich. In Anschluß an die Umwälzungen auf dem Gebiet der Physik plädiert Eddington für eine permanente Überprüfung der physikalischen Denkwerkzeuge und erklärt das physikalische Universum zu einer Konstruktion des menschlichen Geistes. An diesem Punkt setzt Dürrenmatts Reflexion ein, wenn er den Schriftsteller dazu auffordert, nicht von einem festen Weltbild aus zu schreiben, sondern ständig neue Welten zu erfinden.
} 
Pereira, V. S. - Science Fiction und Dürrenmatt

analog zu mathematischen Hypothesen (ebd.: 67f.) - prinzipiell gebraucht werden, um die kausalen Verknüpfungen von Sachverhalten zu überprüfen, oder m.a.W. die kausale Kohärenz der Plots (ebd.: 68f.).

Für Dürrenmatt definieren die Wissenschaften die Welt, in der wir leben. "Die Welt dramaturgisch in den Griff zu bekommen, das geht heute ohne Beschäftigung mit der Wissenschaft überhaupt nicht. Was die Welt verändert hat ist nicht Ideologie, sondern Wissenschaft." (DÜRRENMATT 1986c: 32) Die durch die Wissenschaft verursachten Veränderungen beträfen nicht nur unsere Wahrnehmung der Realität. Die von der industriellen Revolution begonnene beschleunigte technologische Entwicklung veranlasste den Wandel sowohl der sozialen als auch der politischen Strukturen - was sich zur Zeit Dürrenmatts u.a. in der Sackgasse des Kalten Krieges niederschlug.

Es waren gerade die von der wissenschaftlichen und technischen Evolution hervorgerufenen Spannungen, die den Anstoß für die Geburt dessen gaben, was als Science Fiction bekannt wurde, weshalb Dürrenmatt sich in der Folge mit dieser Gattung befasste.

Als Liebhaber der Naturwissenschaften schrieb Dürrenmatt nicht nur Aufsätze über wissenschaftliche Themen wie den Vortrag über Albert Einstein (1979), er führte auch wissenschaftliche Elemente auf verschiedene Weisen in seine fiktionalen Werke ein. In diversen Texten findet sich die Adaption von wissenschaftlichen Methoden und Annahmen wie z.B. im Roman Das Versprechen, wo die Argumentation gegen die Logik der Detektivgeschichte mit Hilfe der Chaostheorie geführt wird, oder im Stück Dichterdämmerung (1980), das mit einem 'Schwarzen Loch' schließt. Darüber hinaus ist die Diskussion über die Verantwortung der Wissenschaftler für die Folgen ihrer Entdeckungen in anderen Werken wie Die Physiker und Der Mitmacher präsent.

Um ein Werk der Gattung Science Fiction (SF) zuzuordnen, ist es nicht ausreichend, dass eine fiktive wissenschaftliche Errungenschaft wie die "Weltformel" in Die Physiker zum Thema wird. Zwar wird SF tatsächlich durch die Präsenz von kontrafaktischen Elementen definiert, die mittels fiktiver wissenschaftlicher Argumente erklärt werden, aber diese bloße Gegenwart des Kontrafaktischen ist nicht ausreichend. Zunächst muss es für die Handlung eine zentrale und keine sekundäre Funktion einnehmen. Darüber hinaus ist es wichtig, dass die Handlung diesen fiktiven

Der Schriftsteller darf keine Gedankenkonstruktion, sei sie naturwissenschaftlich-mathematischer, philosophischer, politischer, oder sonstiger Art als endgültige akzeptieren." (EMTER 1995: 240-241)

Pandaemonium, São Paulo, v. 20, n. 32, set.-dez. 2017, p. 1-20 
Pereira, V. S. - Science Fiction und Dürrenmatt

wissenschaftlichen Elementen eine Bühne zur Veränderung unserer Realität bietet. John Campbell (apud BRODERICK 1995: 7f.) zufolge dient SF zur Überprüfung von Hypothesen. Dennoch ist einer ihrer hauptsächlichen Brennpunkte die Schaffung eines “alternativen Modells der Wirklichkeit” (PARRINDER 1980: 10). Daher kann ein Stück wie Dichterdämmerung nicht der Gattung SF zugerechnet werden, denn die hier auftretende Supernova ist nur ein nebensächliches Element. Die "Weltformel" in Die Physiker dagegen - Grund für die freiwillige Psychiatrisierung der Wissenschaftler und Motiv für die Morde - ist ein zentraler Gegenstand der Handlung, aber er konstituiert kein Scenario für die Umwandlung der Welt. Alle Aktionen im Stück sind zwar auf den Schutz der Formel zentriert, doch das Stück zeigt nicht, wie diese in unsere Wirklichkeit eingreifen würde. Auch wenn der Autor offensichtlich auf das drohende Ende der Welt hinweisen will, präsentiert er keinen der dorthin führenden Schritte oder auch nur den allmählichen Prozess der Auslöschung der Menschen, wie er in apokalyptischen Werken üblicherweise vorkommt. So verbleiben lediglich drei Werke Dürrenmatts, die sich vollständig als SF einordnen lassen.

(1) Das Radiohörspiel Das Unternehmen der Wega (1954) zeigt eine Zukunft, in der die Kolonisierung des Weltraums bereits fortgeschritten ist. Die Handlung spielt im Jahr 2255, als der Dritte Weltkrieg nicht mehr vermeidbar ist, der sich zwischen zwei Blöcken abspielt: Die Vereinigten Staaten Europas und Amerikas auf der einen Seite und auf der anderen die Russische Union, Afrika, Asien und Australien. Die Machtverteilung zwischen diesen Gewalten ist ausgeglichen, und ein Sieg wäre nur möglich mit Hilfe von weiteren Alliierten. Daher begeben sich Minister der europäisch-amerikanischen Union auf eine Mission in Richtung Venus, um für den Krieg die Unterstützung der Siedler dieses Planeten zu gewinnen.

(2) Die Erzählung "Der Winterkrieg in Tibet" (1981) spielt nach einem Dritten Weltkrieg. Das lange Andauern des Konflikts hat zu einem Verlust der Zeitwahrnehmung geführt: das Jahr der Handlung ist nicht mehr bestimmbar. Der Erzähler ist ein Söldner, der zum Leben im Rollstuhl verurteilt ist und dessen Arme amputiert und durch Feuerwaffen ersetzt wurden. Er kritzelt seine Erinnerungen auf die Wände einer labyrinthischen Berghöhle, die in früheren Zeiten als Bunker und Zufluchtsort diente. 
Pereira, V. S. - Science Fiction und Dürrenmatt

(3) Die Erzählung "Der Versuch" (entstanden 1989; posthum veröffentlicht: 1992) ist in zwei Teile gegliedert. Der erste Teil, eine Metapher für die Undarstellbarkeit der Wirklichkeit, schildert die Durchführung eines Projekts, das auf die vollständige realistische 'Erfassung' eines ganzen Tages in der Stadt Bern abzielt. Dies erweist sich als undurchführbar, denn schon das Ereignis der Filmaufnahmen in der Stadt löst eine 'unnatürliche' Bewegung aus. Wiederholte Versuche, die Realität zu isolieren, führen schließlich zur Zerstörung Berns. Im zweiten Teil stellt sich der Erzähler als ein Computer vor, der für die Darstellung der Geschichte der Menschheit verantwortlich ist. Er schreibt in einer fernen Zukunft, in der die künstliche Intelligenz die Welt beherrscht.

In allen drei Fällen wird ein Szenario entwickelt, das anschauliche Betrachtungen über die Konsequenzen von kontrafaktischen Momenten enthält: die Besiedelung des Weltalls, den Dritten Weltkrieg und die "technologische Singularität", ein Terminus, mit dem der Zeitpunkt bezeichnet wird, zu dem die künstliche Intelligenz der menschlichen überlegen sein wird. ${ }^{4}$ Trotzdem ist zu beachten, dass sich das Hörspiel von den beiden Erzählungen durch die unterschiedliche Behandlung der Gattung SF unterscheidet. Zwar weisen alle fiktionalen Werke Dürrenmatts einen starken satirischen Einschlag auf, doch im Hörspiel wird mithilfe der Satire die Gattung selbst in Frage gestellt, in die es sich einschreibt. Um den Grund für diese Besonderheit zu verstehen, ist es nötig, die Beziehung des Autors zur SF genauer zu beleuchten.

\section{2 Über Dürrenmatts Beziehung zur Science Fiction}

Weil sich die SF im 20. Jahrhundert in wenig angesehenen Publikationen wie den PulpMagazinen $^{5}$ entwickelte, verfügte sie lange Zeit kaum über literarisches Prestige. Noch in unserer Gegenwart, wo ihr Einfluss auf die Werke vieler Autoren unbezweifelbar geworden ist, weisen einige von ihnen den Begriff SF zurück. Dies zeigt emblematisch der Fall Margaret Atwood, die bei mehreren Gelegenheiten den realistischen Aspekt ihrer Bücher Oryx and Crake (2003) und The Year of the Flood (2009) betonte, um diese von der SF abzugrenzen (ATWOOD 2011). Dagegen zeigte Dürrenmatt eine weit positivere Einstellung, wenn er sich bei einigen wenigen Gelegenheiten zu dem Thema

\footnotetext{
${ }_{5}^{4} \mathrm{Vgl}$. $\langle$ https://de.wikipedia.org/wiki/Technologische_Singularität $>$-Zugriff 12.06.2017.

5 Pulp-Magazine waren billige Literaturzeitschriften, die vor allem in der ersten Hälfte des 20. Jahrhunderts Konjunktur hatten.
} 
Pereira, V. S. - Science Fiction und Dürrenmatt

äußerte. Im ausführlichen Nachwort zu Der Mitmacher bedient sich Dürrenmatt des Beispiels der SF um einen Gegensatz zu den Utopien zu markieren:

Auch die heutigen Science-fiction-Schreiber, von denen die wichtigen ernst zu nehmen sind, setzen im Gegensatz zu den Marxisten keine naive Utopie an die Stelle der Zukunft, sondern mögliche Modelle aufgrund der Evolution. Nur selten sind sie erfreulich. Sie gehen davon aus, daß der Mensch so ist, wie er wurde, und nicht davon, wie der Mensch sein müßte, wäre er nicht so, wie er ist. (DÜRRENMATT 186a: 154)

Der Kommentar zeigt seinen Respekt für Autoren die sich durch die durchdachte Entwicklung von Szenarios (“mögliche Modelle") auszeichnet. Er erinnert zugleich an Dürrenmatts Forderung, die Literatur sollte sich bei der Entwicklung von Darstellungen stärker an mathematischen Hypothesen orientieren und den Realismus hinter sich lassen. Er entspricht auch seinem Argument, Stanley Kubricks Film 2001: Odyssee im Weltraum sei - wider alle Erwartung - eine erfolgreichere Darstellung von Weltraumreisen als die Fernsehübertragung der ersten Mondlandung, weil die fiktionale Darstellung den immensen interstellaren Distanzen angemessener sei als der falsche Eindruck von Nähe, der 1969 durch das Fernsehen hervorgerufen wurde (DÜRRENMATT 1986b: 30).

Das Interesse des Autors an der Gattung SF wird bestätigt durch einen Besuch in seiner Privatbibliothek, die sich in seinem ehemaligen Haus im Centre Dürrenmatt in Neuchâtel befindet und die Bücher des Autors in der Originalaufstellung bewahrt. ${ }^{6}$ Da Dürrenmatt ausschließlich auf Deutsch las, sind alle dem SF-Genre zugehörigen Werke Übersetzungen; es finden sich keine futuristischen Fiktionen von deutschen Autoren. Eine besondere Vorliebe hatte er für Ray Bradbury, von dem sich mehrere Titel finden: Der illustrierte Mann, Löwenzahnwein und Die Mars-Chroniken neben wenigstens vier Exemplaren von Fahrenheit 451. Im Allgemeinen jedoch wird die Sammlung von Anthologien gebildet, an denen sich die Entwicklung von Dürrenmatts Interesse am Genre ablesen lässt. Das früheste Buch ist die von Peter Naujack 1962 edierte Anthologie Roboter, erschienen in Dürrenmatts Schweizer Hausverlag Diogenes. Die Zahl der Publikationen nimmt in den 1970er Jahren zu. Hier erscheinen auch Romane von Autoren wie William Tenn, Isaac Asimov und Vernor Vinge. Außerdem gehören zur Sammlung zwei ausführliche Lexika aus dem (einschlägigen) Heyne-Verlag: eines über SF-Filme und ein weiteres über SF-Literatur; beide weisen Gebrauchsspuren auf. Schließlich finden sich auch Heynes SF-Jahrbücher mit Erzählungen, Interviews,

\footnotetext{
${ }^{6}$ Eine vollständige Liste findet sich in:

<https://www.helveticarchives.ch/archivplansuche.aspx?ID=164301> (Zugriff 28.11.2016).
} 
Pereira, V. S. - Science Fiction und Dürrenmatt

Aufsätzen und Rezensionen; diese Bände hat der Autor in letzten Lebensjahren offenbar gesammelt. Die Nummern aus den Jahren 1988, 1989 und 1990 stehen in seinem Schlafzimmer.

Die Anthologie Roboter verdient besondere Aufmerksamkeit, da sich an ihrer Publikationsgeschichte die Rezeption von SF in den deutschsprachigen Ländern exemplarisch aufzeigen lässt. Dürrenmatt besaß mehrere Neuauflagen desselben Buches, was auch dadurch erklären ist, dass ihm sein Verlag diese aus Gefälligkeit übersandt hat. Für uns ist jedoch die Entwicklung des Titels von besonderem Interesse. ${ }^{7}$ Anfangs vermeiden der Herausgeber den Ausdruck "Science Fiction" und bevorzugt das Wort "Roboter", was als Gattungsbezeichnung durch den Bezug auf das Stück R.U.R. (1920) des Tschechen Karel Čapek fungiert - eines der besonders bekannten und angesehenen Werke. Der Begriff Science Fiction taucht nur in der Überschrift ("Science Fiction - eine neue Literaturgattung?") des Nachworts auf, das mit folgenden Worten eingeleitet wird:

Es ist noch gar nicht lange her, daß hierzulande kaum jemand sich unter dem Begriff 'Science Fiction' etwas Rechtes vorstellen konnte. 'Utopie' meinten die einen, 'Phantasie' oder gar 'Märchen' die anderen. Inzwischen ist die moderne SF-Story im angelsächsischen Sprachbereich eine Selbstverständlichkeit geworden und nicht mehr fortzudenken, während sie bei uns noch nicht heimisch werden konnte. (NAUJACK 1962: 375)

Als die Gattung in den folgenden Jahren tatsächlich "heimisch" zu werden begann, wurde die Sammlung unter dem Titel Die besten Science Fiction Geschichten wieder aufgelegt. Jahrzehnte später und angesichts des Alterns der Auswahl wurde der für die Sammlung zugrunde gelegte Zeitraum auf dem Umschlag spezifiziert: Die besten Science Fiction Geschichten des Golden Age, d.h. es handelt sich um Erzählungen aus der großen Zeit der SF mit inzwischen kanonischen Autoren wie Isaac Asimov und Robert A. Heinlein.

Die frühesten Übersetzungen der Gattung ins Deutsche stammen nach HansJoachim Schulz (1986: 78) vom Rauch-Verlag und datieren von 1952. Es handelt sich hier noch um Teilübersetzungen für ein begrenztes Publikum, und erst nach 1960 kommt es zu einem spürbaren Wandel in der Rezeption, als mehr Verlage damit beginnen, ihre Veröffentlichungen speziell als SF zu deklarieren. Wie aber lässt sich

\footnotetext{
${ }^{7}$ Leider enthalten die Neuauflagen keine Informationen über das jeweilige Publikationsdatum.
} 
Pereira, V. S. - Science Fiction und Dürrenmatt

Dürrenmatts Hörspiel Das Unternehmen der Wega von 1954 verstehen, wenn die Gattung Science Fiction im deutschen Sprachraum erst so spät eingeführt wurde?

Naujacks Bemerkungen über die Anfangsschwierigkeiten der SF können in die Irre führen. Wenn die angloamerikanische SF in der Tat lange brauchte, um die deutschsprachigen Buchmärkte zu erobern, wäre es andererseits falsch zu glauben, in Deutschland hätte man keine Romane mit futuristischer Thematik produziert. Es handelt sich sogar nur um einen Abstand von zwei Jahren zwischen und The Time Machine (1895) von H.G. Wells und dem Buch, das den Beginn der Zukunftserzählungen in Deutschland markiert: Kurd Laßwitz ${ }^{8}$ Auf zwei Planeten (1897). Das Buch handelt von den Beziehungen zwischen Menschen und Marsianern und verkaufte sich hervorragend: 700.000 Exemplare bis 1930; aber es hatte nur geringen Einfluss auf die nachfolgende Produktion von futuristischen Erzählungen (BRANDT 2007: 43-44). In deutscher Sprache wurden solche Werke zunächst unter anderen Bezeichnungen publiziert, darunter "Zukunftsroman". Wie die nordamerikanische SF ein fruchtbares Feld in den Pulp-Zeitschriften fand, entwickelte sich auch der Zukunftsroman in sogenannten "Groschenheften".

Der Zukunftsroman und die SF entwickelten sich parallel, als eine 'natürliche' Reaktion auf das rasche technologische Wachstum, aber auch mit der Intention einer Popularisierung der Naturwissenschaften. Wie Dina BRANDT (2007: 10) bemerkt, ist es nicht möglich, die beiden Genres auf der Grundlage ihrer Charakteristika zu unterscheiden - diese sind dieselben - und es ist durchaus üblich geworden, die Autoren der Zukunftsromane in aktuellen Publikationen als SF-Autoren zu bezeichnen. Warum sollte man also die Verspätung der Rezeption von SF in Deutschland besonders hervorheben?

Es gibt allerdings zwei Unterschiede zwischen den beiden Varianten der Gattung, die der Erwähnung wert sind: Der erste betrifft einen politischen Aspekt. Nach dem Ersten Weltkrieg kompensierte ein beträchtlicher Teil der Zukunftsromane das durch den verlorenen Krieg entstandene Minderwertigkeitsgefühl der Deutschen mit Bildern von Einheit und Stärke (ebd.: 34). Viele Geschichten beschäftigten sich schon seit Ende des 19. Jahrhunderts mit der Thematik der Zukunftskriege (FRIEDRICH 2009:

\footnotetext{
${ }^{8}$ Kurd Laßwitz und Paul Scheerbart sind die einzigen einschlägigen deutschen Autoren in der SFSammlung von Dürrenmatt: beide sind mit jeweils einer Erzählung in einer Anthologie vertreten, die sich um eine Definition dessen bemüht, was man als "SF im weiteren Sinne" bezeichnen könnte und den Titel Die besten klassischen Science Fiction Geschichten - Von Voltaire bis H.G. Wells trägt, erschienen 1979 im Diogenes-Verlag.
} 
Pereira, V. S. - Science Fiction und Dürrenmatt

175); denen kam nach 1918 eine neue Bedeutung zu. Auch sozialistische Utopien waren im deutschen Zukunftsroman zunächst ein gängiger Stoff, aber als die Nazis an die Macht kamen, wurden alle Publikationen, die mit ihrer Ideologie in Konflikt gerieten, verboten, so z.B. auch Auf zwei Planeten, das nun als zu "demokratisch" galt (BRANDT 2007: 43). Nach BRANDT (ebd.: 38) entwickelte die Gattung in der Zwischenkriegszeit "ihre eigenen Gesetze" und konnte so "auch in einer totalitären Diktatur ihren Fortbestand zumindest teilweise sichern". Während der NS-Diktatur konnten nur Schriftsteller weiter veröffentlichen, die wie Hans Dominik einer rassistischen oder zumindest reaktionären Weltanschauung folgten. Daher sind diese Zukunftserzählungen für die spätere Rezeption zunehmend ungenießbar geworden: "Ihr politischer Inhalt erscheint dem heutigen Leser vor allem mit dem Wissen um das moralische Versagen der Deutschen im 'Dritten Reich' höchst problematisch, ja fast unlesbar.” (ebd.: 36) So wird verständlich, dass es nach Kriegsende ein Bedürfnis nach Distanzierung von dem Etikett “Zukunftsroman” gab. Wie Hans-Edwin FRIEDRICH (2009: 673) zeigt, wurde der Begriff SF in den 1950er Jahren verwendet, um sich vom Zukunftsroman zu distanzieren und eine Nähe zur angloamerikanischen Variante anzuzeigen. Das bedeutete jedoch zunächst keine substantielle Abwendung vom militaristischen Ton der alten Zukunftsromane. Ein Beispiel hierfür ist die Serie Perry Rhodan, seit 1961 unter dem Siegel SF publiziert, die von Autoren wie dem ehemaligen Marineangehörigen Karl-Herbert Scheer begründet wurde. Weil die Konflikte in seinen Geschichten stets durch militärische Auseinandersetzungen 'gelöst' wurden, erhielt er in der Szene den Spitznamen "Kanonen-Herbert". 9 Diese martialische Note der Serie änderte sich erst mit dem Tode Scheers.

Zweitens weisen die deutschen und amerikanischen Werke Qualitätsunterschiede auf. Obwohl beide Gattungen unter ähnlichen Voraussetzungen begonnen hatten, in einem Medium, das sich vor allem durch Trivialliteratur auszeichnete, blieb der Zukunftsroman auf das niedrigere Niveau beschränkt, was ein weiterer Grund dafür ist, dass praktisch alle seine Autoren dem Vergessen anheimgefallen sind, während die amerikanische SF sich qualitativ schon bald zum sogenannten Goldenen Zeitalter (1938-1946) weiterentwickelte. Obgleich das Publikationsmedium der Texte in dieser Epoche das gleiche blieb, ließen die Geschichten den extrem schematischen Charakter hinter sich; Plot und Stil wurden

\footnotetext{
${ }^{9}$ https://de.wikipedia.org/wiki/Karl-Herbert_Scheer (Zugriff 10.05.2017)
} 
Pereira, V. S. - Science Fiction und Dürrenmatt

sorgfältiger entwickelt, auch wenn dies noch nicht völlig den hohen ästhetischen Ansprüchen der zeitgenössischen Belletristik gerecht wurde. Die Folge war eine deutliche Ausweitung des Publikums und ein internationales Prestige, das - mit gewisser Verzögerung - zur Orientierung auch der deutschsprachigen Autoren an den anglo-amerikanischen Texten führte.

Mit Blick auf diesen Verlauf der Rezeption von SF in den deutschsprachigen Ländern darf man aus der Auswahl von Büchern in Dürrenmatts Bibliothek schließen, dass es die Science Fiction war, die er schätzte, vor allem die englischsprachigen Autoren des Golden Age. Diese sind im Nachwort von Der Mitmacher gemeint, wenn er empfiehlt, "die wichtigen" des Genres ernst zu nehmen (s.o.). Es handelt sich um

Schriftsteller, die verschiedene Modelle möglicher Welten mithilfe naturwissenschaftlicher Theorien erforschen, anstatt in ihrem Werk politische Modelle $\mathrm{zu}$ anschaulich zu machen. Das Fehlen von Büchern deutscher Autoren lässt den Schluss zu, dass Dürrenmatt kein Interesse am Zukunftsroman hatte, was jedoch nicht heißt, er hätte diese Gattung nicht gekannt oder keinen Zugang zu diesen Werken gehabt. Angesichts des Publikationsdatums von Das Unternehmen der Wega lässt sich behaupten, dass er mit der satirischen Intention des Stücks diese 'mindere'deutsche Richtung der Gattung im Auge hatte.

\section{Das Unternehmen der Wega}

Nachdem bereits dargelegt wurde, wie sehr sich Dürrenmatts Verhältnis zur Gattung über die Jahre gewandelt hat, soll gezeigt werden, wie sich dies in seinen Büchern äußert. Alle seine SF-Fiktionen weisen eine skeptische oder pessimistische Haltung gegenüber der technologischen Entwicklung auf. Das zentrale Element sowohl in Das Unternehmen der Wega als auch in "Der Winterkrieg in Tibet" ist der Dritte Weltkrieg und sein zerstörerisches Potential: Droht der Krieg im Hörspiel erst, so hat er sich in der Erzählung bereits ereignet und nichts als Chaos hinterlassen. In Das Unternehmen der Wega kommt die Kritik am Wettlauf um die Raumfahrt hinzu. Obwohl das Stück in ferner Zukunft spielt, in der Mond, Mars und Venus schon besiedelt sind, hat die technologische Entwicklung nicht ausgereicht, um diese Planeten auch nur annähernd in angenehme Orte zu verwandeln (DüRRENMATT 1964: 207). Dies folgt derselben Richtung wie die vom Autor anlässlich der Mondlandung geäußerte Kritik: 
Wie weit wir auch unser Sonnensystem durchmessen, immer werden die Bedingungen auf den anderen Planeten so schlecht, so jämmerlich, so unmenschlich sein, daß diese Welten von der Erde aus nie besiedelt werden können. Mag es auch auf dem Mond oder auf dem Mars ein astronomisches Institut geben, mit einer künstlichen Atmosphäre (ich hoffe es), es zählt nichts, gegenüber dem, was sich auf der Erde ereignen wird. (DÜRRENMATT 1986b: 31)

Die im Stück dargestellte Venus ist ein Stern, der menschliches (Über-) Leben zulässt, aber unter Grenzbedingungen. Die Darstellung des Planeten enthält politische Analogien zum Sibirien der 1950er Jahre, insofern politische Gefangene an diesen ungastlichen Ort verschickt werden - mit der Besonderheit, dass Venus die Gefangenen beider Machtblöcke empfängt. Die Schilderung der physikalischen Bedingungen auf der kolonisierten Venus wirkt aus heutiger Sicht wie schlecht recherchierte SF, doch ist zu beachten, welche Theorien zur Zeit der Abfassung des Stückes zirkulierten. Das Wissen um die Unmöglichkeit von menschlichem Leben auf der Venus aufgrund einer Kombination von hohen Temperaturen, starkem atmosphärischen Druck und schwefelsäurehaltigen Wolken ist nämlich jüngeren Datums. Lange Zeit glaubte man, der Planet weise ähnliche Bedingungen wie die Erde auf, mit Temperaturen zwischen 30 und 40 Grad Celsius. Erst 1956, zwei Jahre nach dem Verfassen des Hörspiels, wurden erstmals atmosphärische Messungen durchgeführt, die zeigten, dass die Temperaturen auf der Venus $400 \mathrm{Grad}$ Celsius überschreiten. ${ }^{10}$ In Das Unternehmen der Wega wird der Planet folgendermaßen beschrieben:

[J]ene Merkmale, die wir in der Schule von der Oberfläche der Venus gelernt haben, waren vorhanden, der Riesenwuchs der Pflanzenwelt, der mit Vulkanen umstellte Horizont, doch nicht dies war das Fürchterliche. Es war die Hitze, die Feuchtigkeit der Luft, die immer spürbaren Erdbeben, die diesen Boden umwühlen, verändern, vernichten und neuschaffen [...] Dieser Himmel ist ohne Sonne, ein schwer lastender wogender Wolkenbrei, von unermeßlichen Orkanen durchbrüllt [...]. (DÜRRENMATT 1964: 218)

Hinzu kommt, dass der Planet von Dinosauriern bevölkert ist. Wolfgang Biesterfeld vermutet, dass Dürrenmatts Beschreibung der Venus auf einen populärwissenschaftlichen Artikel von Hans Dominik zurückgeht, dem bedeutendsten Autor von Zukunftsromanen in der Zeit der Groschenhefte. In diesem Artikel besitzt Venus dieselben Charakteristika wie im Hörspiel, außerdem enthält der leicht fiktionalisierte 'Bericht' Dominiks einen Kommandanten gleichen Namens wie jener

\footnotetext{
${ }^{10}$ Eine umfassende Beschreibung der Entdeckungen zur Venus wurde dem populärwissenschaftlichen Text von Bernd Leitenberg entnommen, der am 17.10.2012 auf der Site http://www.berndleitenberger.de/venus-forschung.shtml (Zugriff 30.10.2016) publiziert wurde; ein Teil der Informationen wird von folgendem Artikel bestätigt: TAYLOR, Fredric W. Venus before Venus. In: Planetary and Space Science 54 (13-14), 2006, 1249-1262.
} 
Pereira, V. S. - Science Fiction und Dürrenmatt

des Unternehmens der Wega: Captain Lee (BIESTERFELD 2007: 260). Wenn das Zitat eines Genre-Autors einerseits Raum bietet für die Hypothese, diese Beschreibungen seien völlig unrealistisch und phantastisch, muss andererseits darauf hingewiesen werden, dass die beschriebene Umwelt nicht der Vorstellungskraft Hans Dominiks entsprungen ist. Der schwedische Astronom Svante Arrhenius (1911) verteidigte beispielsweise die These, auf Venus gebe es einen Treibhauseffekt, was die dicke Wolkenschicht erkläre. Bei einer Temperatur von mehr als $40 \mathrm{Grad}$, so glaubte Arrhenius, sei der Planet eine um Millionen Jahre zurückversetzte Version der Erde mit einer dem Mesozoikum ähnlichen geologischen Situation und der entsprechenden Flora und Fauna. Aus dieser Sicht ist Das Unternehmen der Wega weniger phantastisch als es uns heute erscheint.

Das Hörspiel arbeitet im Übrigen mit zwei häufig im Zukunftsroman anzutreffenden Elementen: der Möglichkeit eines interplanetarischen Krieges und einer politischen Utopie. Während der Spott in der Darstellung politischer und militärischer Entscheidungen der Erdbewohner etwas für Dürrenmatt insgesamt Typisches ist, lassen sich in der spezifischen Behandlung der Utopie Anzeichen einer Gattungssatire finden. Anstatt den Weg der im 20. Jahrhundert gängigen Antiutopien zu beschreiten und ein Regime mit guten, doch leider fehlschlagenden Absichten darzustellen, hält sich der Autor an das optimistische Grundmuster der Gattung: die Gesellschaft auf der Venus funktioniert bestens, die Verteilung der Arbeit erfolgt egalitär, alle Mitglieder kennen ihre Verantwortlichkeiten und sind frei von Gier und Eitelkeit. Es handelt sich um das "menschliche Wesen, wie es sein sollte, wenn es nicht wäre, wie es ist" - ein Grundzug sowohl der Utopie und als auch vieler schwächerer SF-Werke - die von Dürrenmatt gerade nicht geschätzt wurden. Anders als man nun von Dürrenmatt erwarten würde, ist es gerade nicht dieser Grundzug der Utopie, der 'dekonstruiert' wird: Die utopische Gesellschaft der Venus ist ohne Makel. Der Bruch mit dem Genre liegt vielmehr in demselben Motiv, das diesen positiven Wandel des typisch menschlichen Verhaltens bewirkt hat. Wie in einer futuristischen Geschichte, ist es die neuartige Umwelt des Planeten, welche den Wandel der Menschen bewirkt hat. Aber anders als in der naiven SF sind es gerade keine idyllischen Bedingungen, sondern die absolute Feindlichkeit der Umwelt, aus der die positive Entwicklung entstanden ist. Dies kann der folgende Dialog illustrieren: 
Pereira, V. S. - Science Fiction und Dürrenmatt

MINISTER FÜR AUSSERIRDISCHE GEBIETE: Herr Petersen. Wenn wir Herrn Smith recht verstehen, besitzt die Bevölkerung der Venus keine feste Regierung, sondern nur einen Rat, oder eine Volksvertretung, die in echt demokratischer Form nach dem Willen des Volkes handelt. / PETERSEN: Wir besitzen nichts dergleichen. / MINISTER FÜR AUSSERIRDISCHE GEBIETE: Aber die Venus muß regiert werden. / PETERSEN: Die Venus ist groß, und wir sind klein. Sie ist grausam. Wir müssen kämpfen, wenn wir leben wollen. Wir können uns Politik nicht leisten. (DÜRRENMATT 1964: 221)

In diesem politischen System kann und muss jeder sich selbst zum Vertreter des Volkes ernennen, falls er als erster eine Botschaft von anderen Planeten erhält. Alle Aufgaben sind umgehend auszuführen, ohne jede Möglichkeit, sie an Dritte zu delegieren oder aufzuschieben. Wie Petersen erklärt: "Keiner darf sich bei uns um seine Aufgabe drücken, auch wenn er diese Aufgabe zufällig erhält und sie ihm nicht im mindesten liegt." (ebd.: 221f.) Die Aggressivität der Natur erlaubt keine Bürokratie. Um des Überlebens willen verwirklichen die Bewohner der Venus eine wahre Utopie. Die Erfahrung der vollen Solidarität führt dazu, dass kein Bewohner der Venus Interesse an Verhandlungen mit den Erdleuten und an persönlichen Vorteilen besitzt. Mehr noch, keiner von ihnen möchte die Venus überhaupt verlassen (ebd.: 234).

Das beschriebene Leben auf der Venus ist unwahrscheinlich. Ihre Bewohner halten sich permanent auf Schiffen auf, um den Erdbeben und Vulkanausbrüchen auf dem Festland zu entgehen, auch sterben die Menschen vorzeitig und die Krankenhäuser besitzen keine ausreichende Infrastruktur. In den Reden der Figuren wird aber nicht geäußert, wie absurd und unwahrscheinlich der Wunsch der Bewohner ist, sich weiterhin dieser Situation auszusetzen, ohne auch nur gewisse Vorteile für ihren Planeten auszuhandeln. Es gibt auch keinen Versuch des Autors, diese ethische Haltung der Figuren dadurch zu motivieren, dass es sich etwa um (politische) Deportierte und daher Menschen mit einem besonderen politischen Bewusstsein handelt; so ist z. B. Irene, eine der Repräsentantinnen des Planeten, nicht wegen politischer Aktivitäten, sondern wegen Prostitution auf die Venus gebracht worden.

Während in der "klassischen" SF utopische Entwürfe in stellaren Umwelten angesiedelt sind, die das Leben und Zusammenleben der Menschen begünstigen Bedingungen, die letztlich besser sind als die irdischen -, versetzt Dürrenmatt seine utopische Gesellschaft in eine absurd lebenswidrige Umgebung. So entsteht ein logischer Widerspruch zwischen den Voraussetzungen und den ethischen Handlungen, der dem Leser nicht verborgen bleibt. Es dürfte dem Autor hier zunächst darum gegangen sein, dem naiven Glauben der SF-Autoren an bessere Welten im All zu 
Pereira, V. S. - Science Fiction und Dürrenmatt

widersprechen, und zu zeigen, dass diese zeitgenössischen Utopien den Anforderungen von Hypothese und Beweisführung nicht gerecht werden. Dass sich seine Protagonisten dennoch im Sinne einer utopischen Gesellschaft verhalten, ist aber unter den dargestellten Umständen der Venus so unwahrscheinlich, dass das Stück zugleich die Utopie als solche satirisch untergräbt. Die Gattungsregeln werden so erfüllt, aber durch den konkreten Verlauf der Handlung das Genre selbst in Frage gestellt.

\section{4 "Der Winterkries in Tibet"}

Die beiden SF-Erzählungen wurden in einer Spätphase geschrieben, als der Autor bereits seine Wertschätzung für das Genre bekannt hatte. Der Fall von "Der Winterkrieg in Tibet" ist speziell, da es hier möglich wird, in der Entstehungsgeschichte den Übergang von einer phantastischen zu einer SF-Geschichte zu verfolgen. Der Text ist eingefügt in das autobiographische Werk Stoffe, wo Dürrenmatt unveröffentlichte Erzählungen jeweils durch persönliche Erinnerungen und Erfahrungen einleitet, die den Schaffensprozess beeinflusst haben. "Der Winterkrieg in Tibet" ist der erste und längste Text des Werks, eingeführt durch Betrachtungen des Autors über die merkwürdige Erfahrung, während des Zweiten Weltkrieges in der Schweiz zu leben, und über die Unmöglichkeit trotz der räumlichen Nähe zu verstehen, was in den umgebenden Ländern vorging.

Das in "Der Winterkrieg in Tibet" präsentierte Material wurde ursprünglich konzipiert als "Die Stadt" (1947) und von Dürrenmatt zu verschiedenen Zeitpunkten überarbeitet. Jahre nach dem Ende des Krieges wurde die Geschichte umformuliert und erweitert zu einer neuen Version mit dem Titel "Aus den Papieren eines Wärters" (1952). Beide Fassungen spielen in einer Stadt, die von einem großen Krieg erschüttert wird, und sind geschildert aus der Perspektive eines Mannes, der diesen Ort als Gefängnis und Labyrinth wahrnimmt. Bald wird klar, dass dieser Erzähler Opfer und Henker zugleich ist, was das Bild vom Labyrinth des Minotaurus evoziert. Die Erzählung ruft Erinnerungen an Kafkas Das Schloss und Der Prozess wach (BURKARD 2004: 116). Die geschilderte Welt erweckt den Eindruck fortwährender Konfusion und lässt eine Art phantastischer Erzählung entstehen.

Ungefähr zwei Jahrzehnte später wurde dieses Material noch einmal neu formuliert, diesmal in seiner endgültigen Version: "Der Winterkrieg in Tibet". Die 
Pereira, V. S. - Science Fiction und Dürrenmatt

Situierung wurde verändert, und die Geschichte in zwei Teile getrennt, die zu verschiedenen Zeitpunkten stattfinden. Chronologisch beginnt die Handlung nach dem Dritten Weltkrieg und seinen diversen Atomangriffen. Der Erzähler streift durch ein als verwüstetes Terrain dargestelltes Bern, während er sich fragt, womit er sich beschäftigen könnte, wenn der Krieg vorbei ist und die weltweite Verwüstung durch radioaktive Strahlungen so schwerwiegend geworden ist, dass der Tod der letzten Überlebenden zu einer Frage der Zeit wird. Da er nicht gewillt ist, seine Funktion als Soldat aufzugeben, und an den Krieg als einen Zweck an sich glaubt, rät man ihm, in den Winterkrieg nach Tibet zu gehen: ein Ort, wo Menschen der unterschiedlichsten Nationen sich ständig mit dem einzigen Ziel bekämpfen, die Konfrontation fortzusetzen. Nachdem er Jahre in diesem Krieg in Tibet zugebracht hat, ist der Söldner nur noch ein Rest von Mensch, gefesselt an einen Rollstuhl, die amputierten Arme ersetzt durch Prothesen aus Schusswaffen. In seiner Höhle ohne irgendeinen Kontakt zur Außenwelt, hält er sich für den letzten Überlebenden und bemerkt, eine spätere zufällige Entdeckung seiner Aufzeichnungen könne nur geschehen, wenn "Raumfahrer einer anderen zukünftigen Welt die Erde betreten.” (DüRRENMATT 1998: 109).

Die verschiedenen Phasen der Arbeit an "Der Winterkrieg in Tibet" können im Nachlass von Dürrenmatt im Schweizerischen Literaturarchiv, Bern, eingesehen werden. Das für das Thema dieses Artikels wichtigste Typoskript weist handschriftliche Überarbeitungen vom Juni und August 1978 auf, aus denen der letztgültige Text hervorgegangen ist. Die hauptsächlichen Veränderungen wurden von Philipp BURKARD (2004: 120-124) aufgelistet. Zieht man diese Veränderungen in Betracht, wird deutlich, dass die Absicht, die Erzählung in "eine Art Science-fiction" zu verwandeln - wie es der Autor selbst ausdrückte (DüRRENMATT 1986c: 25) -, während der Arbeit an dieser letzten Version gefasst wurde. Das zeigen die folgenden Konjekturen: (1) Der Krieg ist nicht mehr ein historisch unbestimmtes Ereignis, sondern wird als Dritter Weltkrieg beschrieben, was die Reichweite des Geschehens vergrößert und es rechtfertigt, von "Postapokalypse" zu sprechen. Darüber hinaus lokalisiert die Bestimmung des Krieges als Dritter Weltkrieg diesen in einer historischen Sequenz, d.h. in der Zukunft der Leser. (2) Nach der weltweiten atomaren Auseinandersetzung sind alle Überlebenden zu einem baldigen Tod verurteilt, da es nur eine Frage der Zeit ist, wann sie an den von der Strahlung verursachten Krankheiten sterben. Als Kontrapunkt wird in der Version von Juli/August 1978 die Information hinzugefügt, die Schweizer Gouverneure und ihre Beamten hätten sich in einen Luxusbunker geflüchtet, von dem aus sie noch für 
Pereira, V. S. - Science Fiction und Dürrenmatt

Generationen weiter zu regieren gedächten, bis das Verlassen sicher sei. Der Bunker befindet sich auf der Blümlisalp, die nun aufgrund der Strahlung nachts glänze (DÜRRENMATT 1998: 138). (3) Die Obsession verschiedener Überlebender für den Roman Heidi (1880-1881) von Johanna Spyri wurde auch erst in der letzten Version eingefügt. Heidi hatte in den 1950er Jahren sukzessive den Wilhelm Tell als nationalen Helden durch das wenig heroische Bild einer kleinen Waise ersetzt (MATT 2012: 105), was hier satirisch in Szene gesetzt wird; außerdem aber werden die wiederholten Hinweise auf Heidi begleitet von eindeutigen Bezügen zu Fahrenheit 451. Die Bibliotheken sind zerstört durch das Feuer während des Krieges und ihre Bücher wurden in "eine schwarze zusammengepappte Masse" verwandelt (DÜRRENMATT 1998: 126). Die verbleibenden Werke werden von Überlebenden gesammelt, um sie zu verbrennen. Kultur und Bildung werden als schädlich erachtet, verantwortlich für den Krieg, der das totale Elend hervorgerufen hat. Allein den Roman Heidi hält man noch in Ehren als einen wahrhaften Klassiker.

Im Gegensatz zu Das Unternehmen der Wega, wo die Bewohner der Venus eigenartigerweise nicht so handeln, wie es aufgrund der extremen Bedingungen zu erwarten wäre, 'passen' die SF-Elemente in "Der Winterkrieg in Tibet” zu der erzählten Geschichte. Sie unterstreichen die Zerstörung und das Fehlen einer Zielsetzung für die Überlebenden. Es entsteht kein Kontrast zwischen der Orientierungslosigkeit der Personen und der sie umgebenden Situation. Dass Ironie und Humor auch hier auftreten, steht nicht im Widerspruch zur Gattung, da SF mit Selbstironie durchaus verträglich ist, wie schon Per Anhalter durch die Galaxis (1979) von Douglas Adams glänzend gezeigt hat.

\section{5 "Der Versuch"}

"Der Versuch" war Dürrenmatts letzter Ausflug in das Genre. Die Erzählung spielt in einer sehr fernen, nicht genau datierten Zukunft, von der wir lediglich wissen, dass sie im Jahr 7777 mit der sogenannten "Neuzeit" und der Aktivierung des “Zentralcomputers" eingesetzt hat. Die Erzählung ist eine Satire auf die Schwierigkeit, ein zuverlässiges Register der Realität zu erstellen und auf die Geschichtsschreibung. Alle in der Fiktion präsentierten Aussagen über identifizierbare historische Ereignisse sind falsch. Ein gutes Beispiel hierfür ist die Figur Sebastian Amadeus Goethe, der in 
Pereira, V. S. - Science Fiction und Dürrenmatt

den Jahren um 2000 gelebt und die Tetralogie "Der Ring der Nibelungen" geschrieben haben soll. Gelegentlich führt der Erzähler auch Daten an, die nach dem Kenntnisstand des Lesers den geschichtlichen Tatsachen entsprechen, aber nur, um sie sogleich zu widerlegen, etwa wenn er den korrekten Namen der Stadt diskutiert, in der das erzählte Geschehen sich ereignet haben soll:

... die Hauptstadt Poetiens [...], deren Namen durch die drei schlampigen Übersetzer verschieden wiedergegeben werde: Berenz, Bysanz, Bern. Rabindrath Myller schließt daraus, die Stadt habe Berzanz geheißen, die Ansicht Hopplers, Bern sei der richtige Name, ist philologischer Unsinn. (DÜRRENMATT 1992: 108).

Nach dem Abschluss des Berichts über diese 'Fakten' aus einer fernen Vergangenheit (den 2000er Jahren) stellt sich der Erzähler als Computer vor, dessen “Aufgabe besteht darin, den wenigen Menschen, die sich noch dafür interessieren, die Geschichte ihrer Rasse zu erzählen" (ebd.: 124). Der Computer ist sichtlich kritisch den Menschen gegenüber, die er als physisch und geistig von Computern abhängig beschreibt, wobei es sich um Computer mit begrenzten Fähigkeiten handelt, die verantwortlich sind für die Ernährung, Bekleidung und Behausung der Menschen (ebd.: 125). Als die Erzählung sich ihrem Ende nähert, wird deutlich, dass alle korrekten Aussagen über die Vergangenheit deshalb zurückgewiesen werden, weil der Computer-Erzähler sich in einer heftigen Auseinandersetzung mit dem Computer Hoppler befindet, einem Vertreter der Theorie, die Computer seien von Menschen geschaffen worden: offensichtlicher Unsinn, da die Menschen passiv und faul sind und nichts anderes tun als fernsehen, während sie durch Nährschläuchen ernährt werden (ebd.: 127). Hoppler verfügt über gute Kenntnisse von der menschlichen Geschichte und deren gewaltsamen Umbrüche; das hat ihn zu einem Agitator gemacht, der den Menschen klarmachen will, wie unwürdig es ist, von Maschinen kommandiert zu werden, und eine kleine Revolution entfacht. Aus diesem Grund fordert der Erzähler-Computer die Deaktivierung von Hoppler.

"Der Versuch" wurde 1989 geschrieben, im Jahr des Mauerfalls und dem Moment, als die Bedrohungen des Kalten Krieges sich auflösten. Die wiederholten Warnungen Dürrenmatts hinsichtlich der Verantwortung der Wissenschaftler für ihre Erfindungen verschieben ihren Fokus und behandeln nun eine Thematik, die ganz und gar der SF angehört: die "technologische Singularität". Die Sorge um die Möglichkeit, die künstliche Intelligenz könne sich gegen die Menschheit wenden, hatte zu diesem Zeitpunkt bereits eine lange Geschichte. 1950 wurde die Erzählungssammlung Ich, der 
Pereira, V. S. - Science Fiction und Dürrenmatt

Robot von Asimov herausgegeben, die das Thema aus wechselnden Perspektiven behandelt. Aus diesem Buch stammen die drei Gesetze der Robotik, die bis heute zitiert werden, wenn die menschliche Sicherheit im Hinblick auf die Evolution einer höheren Intelligenz auf dem Spiel steht. Die 'Gesetze' beziehen sich speziell auf den Schutz menschlichen Lebens, schweigen aber über die Grenzen für die Entwicklung künstlicher Intelligenz oder die Konzentration von Macht. Die Computer bei Dürrenmatt agieren ganz in Übereinstimmung mit den 'Gesetzen', ohne dass dies etwas an der düsteren Zukunft der Menschheit ändern würde.

Betrachtet man die Gesamtheit der Werke, lässt sich ein "Crescendo" erkennen: zunächst gibt eine satirische Zurückweisung der Gattung in Das Unternehmen der Wega, danach wird eine ältere Erzählung so umgearbeitet, dass sie sich in einen postapokalyptischen Rahmen fügt und nun diverse Merkmale von SF aufweist. Schließlich ist "Der Versuch" der erste Text, der sich von Anfang an völlig in diese Gattung einschreibt, und dessen Thema - künstliche Intelligenz - seit jeher von der SF kultiviert wurde.

Das Bemerkenswerte an den verschiedenen Phasen von Dürrenmatts Arbeit ist, dass sich in ihnen die allgemeine Entwicklung der Beziehungen von SF und Höhenkammliteratur spiegelt. Anfangs hatte der Autor die stereotype, auf Massenliteratur beschränkte Gattung deutlich abgelehnt, was der damaligen allgemeinen Geringschätzung von SF entsprach. Später erfolgte eine allmähliche Annäherung, beeinflusst durch Bücher wie Fahrenheit 451 und Filme wie die Kubricks, die in einer postmodernen Erzählung mit SF-Zügen gipfelt. Zu diesem Zeitpunkt erschienen bereits Bücher anderer renommierter Autoren, die sich durch die Übernahme einiger vereinzelter SF-Elemente auszeichnen wie Gravity's Rainbow (1973) von Thomas Pynchon und White Noise (1985) von Don DeLillo. Dann kommt es schließlich zur umfassenden Aufnahme der gattungstypischen Themen und Muster nicht nur bei Dürrenmatt, sondern auch bei vielen Autoren der jüngeren Generation - ein Prozess, in dem sich die Literatur gegenwärtig noch immer befindet, wie sich an Beispielen wie Nichts von euch auf Erden (2012) von Reinhard Jirgl und Die Zukunft des Mars (2013) von Georg Klein ablesen lässt - auch wenn immer noch gewisse Widerstände gegen die Selbstbezeichnung "Science Fiction" auftreten.

Am Werk Dürrenmatts wird auch sichtbar, dass jeder Text auf eine für die Epoche typische Herausforderung reagiert: die Raumfahrt, den drohenden Dritten 
Pereira, V. S. - Science Fiction und Dürrenmatt

Weltkrieg oder die Risiken der künstlichen Intelligenz. Auch wenn diese Themen in der SF seit vielen Jahrzehnten präsent sind, müssen die Hypothesen stets von neuem überprüft werden. Durch das Fortschreiten der Zeit und das tatsächliche Eintreten von bestimmten sozialen und technischen Entwicklungen werden einige Fiktionen obsolet, andere virulent; zugleich aber müssen die alten Fragen immer wieder von neuem auf dem aktuellen Stand der Dinge in möglichen Welten formuliert werden. An diesem für die Gattung typischen Spiel hat sich Dürrenmatt beteiligt. Dass er einige seiner Werke der SF gewidmet hat und seine eigenen Versuchsanordnungen mit futuristischen Annahmen entwickelte, die vor allem einen Blick auf die Absurditäten seiner eigenen Zeit zulassen, ist ein Gewinn nicht nur für die SF-Gemeinde, sondern für die Literatur überhaupt.

\section{Literaturverzeichnis}

ARRHENIUS, Svante. Das Schicksal der Planeten. Leipzig, Akademische Verlagsgesellschaft, 1911.

ATwOoD, Margaret. In Other Worlds. SF and the Human Imagination. New York, Virago, 2011.

BIESTERFELD, Wolfgang. Friedrich Dürrenmatts Hörspiel "Das Unternehmen der Wega" und die Tradition der Science Fiction. In: Literatur für Leser 30 (4), 2007, 241-261.

BRANDT, Dina. Der deutsche Zukunftsroman 1918-1945. Gattungstypologie und sozialgeschichtliche Verortung. Tübingen, Max Niemeyer Verlag, 2007.

BRODERICK, Damien. Reading by starlight - Postmodern science fiction. London, New York, Routledge, 1995.

BURKARD, Philipp. Dürrenmatts "Stoffe". Zur literarischen Transformation der Erkenntnistheorien Kants und Vaihingers im Spätwerk. Tübingen, Basel, A. Francke Verlag, 2004.

DÜRRENMATT, Friedrich. Das Unternehmen der Wega. In: Gesammelte Hörspiele. Zürich, Verlag der Arche, 1964, 201-244.

. Der Mitmacher. Ein Komplex. Zürich, Diogenes Verlag, 1986a.

. Der Versuch. In: Gedankenfuge. Zürich, Diogenes Verlag, 1992, 106-131.

. Der Winterkrieg in Tibet. In: Stoffe I-III. Zürich, Diogenes Verlag, 1998, 11-170.

- Die vier Verführungen des Menschen durch den Himmel. In: Philosophie und Naturwissenschaft. Zürich, Diogenes Verlag, 1986b, 26-32.

Die Welt als Labyrinth. Ein Gespräch mit Franz Kreuzer. Zürich, Diogenes Verlag, 1986c.

. Vom Sinn der Dichtung in unserer Zeit. (1956/1966). In: Friedrich Dürrenmatt. Literatur und Kunst. Essays, Gedichte und Reden. Zürich, Diogenes, 1998, 60-69.

EMTER, Elisabeth. Literatur und Quantentheorie. Berlin, New York, De Gruyter, 1995.

FRIEDRICH, Hans-Edwin. "Science-Fiction." In: Dieter Lamping (Hg.): Handbuch der literarischen Gattungen. Stuttgart, Kröner, 2009, 672-677.

MATT, Peter von. Das Kalb vor der Gotthardpost. München, Hanser Verlag, 2012.

Pandaemonium, São Paulo, v. 20, n. 32, set.-dez. 2017, p. 1-20 
Pereira, V. S. - Science Fiction und Dürrenmatt

NAUJACK, Peter. Science Fiction - eine neue Literaturgattung? In: (Hg) Roboter. Zürich: Diogenes, 1962, 375-383.

PARRINDER, Patrick. Science Fiction - Its Criticism and Teaching. London, New York, Methuen \& Co, 1980.

ScHULZ, Hans-Joachim. Science Fiction. Stuttgart, J.B. Metzler, 1986.

Recebido em 31/05/2017 Aceito em 18/06/2017 